\title{
Aislamiento, identificación y evaluación de la actividad antimicrobiana de metabolitos producidos por Mucor circinelloides (Cepa Nativa SPG 321)
}

\author{
Isolation identification and evaluation of the antimicrobial activity for \\ metabolites produced by Mucor circinelloides
}

Título abreviado: Mucor circinelloides y su actividad antimicrobiana

Aura Patricia Chaparro Pedraza', Silvia E. Campuzano F. ${ }^{2}$

\section{Resumen}

Objetivo. Identificar y evaluar la capacidad antimicrobiana de los metabolitos aislados a partir del proceso de fermentación en una cepa de Mucor circinelloides. Método. En el presente estudio se trabajó la cepa nativa de Mucor circinelloides SPG 321 suministrada por el Grupo de Investigación de Biotrasformación (GIBUJ), con el fin de evaluar su actividad antimicrobiana. La cinética de crecimiento determinó que la ideofase culminó a la hora 264, hora determinada como la finalización de la fermentación. Se separó el micelio del caldo y posteriormente se fraccionaron los extractos con cromatografías de capa fina y columna en sílica gel, eluidas con diferentes relaciones de solventes. Resultados. Los resultados arrojados por la técnica de gases acoplado a masas CG-EM confirman la importancia de Mucor circinelloides en la producción de ácidos grasos insaturados. A partir del micelio se obtuvo un esterol, compuesto M. cB3. La fracción $\mathrm{CHCl} 3$ en biomasa mostró actividad inhibitoria para los microorganismos Gram positivos.

Palabras claves: Mucor circinelloides, ácidos grasos insaturados, actividad antimicrobiana, metabolitos secundarios.

\begin{abstract}
Objective. To identify and evaluate the antimicrobial capacity of the metabolites isolated from the fermentation process in a strain of Mucor circinelloides. Method. For this study it was taken the 321 SPG native strain of Mucor circinelloides provided by GIBUJ. The kinetics growth was made, observing the ending of the idiofase at hour 264, hour taken for the conclusion of fermentation. Mycelium was separated from the broth and then purified with thin layer chromatography and silica gel column, eluted with different relations of solvents. Results. The results given by the technique of coupled gases with GC-MS mass confirm the importance of Mucor circinelloides in the production of unsaturated fatty acids. A sterol was obtained from the mycelium, M.cB3 compound. The fraction $\mathrm{CHCl} 3$ in biomass showed inhibitory activity for Gram-positive microorganisms.
\end{abstract}

Keywords: Mucor circinelloides, unsaturated fatty acids, antimicrobial activity, secondary metabolites.

1. Investigadora del Grupo de investigación BAZERI. Universidad Colegio Mayor de Cundinamarca.

2. Investigadora del Grupo de investigación BAZERI. Universidad Colegio Mayor de Cundinamarca. 


\section{Introducción}

El conocimiento de la biodiversidad, es un tema importante y complejo que abarca desde la variabilidad genética de especies, hasta el conocimiento de las relaciones que se establecen entre los seres humanos y la naturaleza (1). Por la riqueza en recursos naturales de Colombia se debe apoyar la investigación de los mismos, como una herramienta en la búsqueda de nuevos principios activos que aporte al crecimiento económico y desarrollo social del país.

El $40 \%$ de las investigaciones en biodiversidad centran su trabajo en las plantas como grupo taxonómico, $33 \%$ en vertebrados, $21 \%$ en invertebrados y sólo el $14 \%$ en los microorganismos. El conocimiento sobre metabolitos generados por especies nativas es escaso en la actualidad, por eso el particular interés se centra en entender este proceso para la cepa nativa SPG 321 de Mucor circinelloides. La cepa fue recolectada en el páramo de Guasca (2); este es un hongo filamentoso que pertenece a la clase Zygomycetes, al cual se le realizó aislamiento de metabolitos mayoritarios y se evaluó su actividad antimicrobiana y antifúngica.

\section{Materiales y métodos}

\section{Microorganismo}

Se seleccionó la cepa de Mucor circinelloides SPG 321, de la colección de especies del banco de cepas del grupo de investigación de Biotransformación de la Universidad Javeriana GIBUJ, conservada en suelo. Para su activación, se esparcieron granos de suelo sobre agar papa dextrosa (PDA), Agar Czapeck $(\mathrm{Cz})$ y agar extracto de malta (MEA). Se obtuvieron cultivos monospóricos para su identificación.

\section{Identificación}

Teniendo en cuenta las claves taxonómicas de Gilman (3) y Samson y otros autores (4), se realizó la observación macroscópica durante la primera y la segunda semana. Se realizó la identificación microscópica utilizando azul de lactofenol, en diferentes aumentos, se estudió el crecimiento de la cepa en luz azul y se realizó prueba de termotolerancia (5).

\section{Parámetros cinéticos}

A partir de una suspensión de 1×107 conidias/mL, en caldo papa-glucosa-sulfato de amonio (PGS), a $\mathrm{pH} 7.0 \pm 0.2$ y $5 \mathrm{ml}$ se utilizaron 16 erlenmeyer de $250 \mathrm{ml}$ por un periodo de 384 horas, con relación $1 / 5$ de aireación. Se identifican las fases de crecimiento (6).

\section{Proceso fermentativo}

La fermentación en batch se llevó a cabo en erlenmeyer de 5 litros, con un volumen efectivo de trabajo (VET) de $1000 \mathrm{~mL}$. En medio PGS, se alcanzó 20 litros de caldo de cultivo. Cada fermentación se lleva a cabo a temperatura ambiente por 11 días (264 horas) con agitación de 160 rpm. Para la aireación se uso motor ELITE 799 con una salida de aire de $1000 \mathrm{cc} / \mathrm{min}$, conservando la proporción de $1 / 5$ para proporcionar un medio aeróbico al cultivo $(7,8)$.

\section{Extracción de los metabolitos}

Los cultivos de cada fermentación se filtraron al vacío, de esta manera se obtuvo caldo y biomasa. Para el caso del caldo se realizó una extracción líquido-líquido con AcOEt, se concentró al vacío en rota vapor BUCHI-RE1 11 y se obtuvo un extracto crudo de caldo. La biomasa seca se maceró y percoló con diferentes solventes en técnica Soxhlet (9). Los extractos totales se fraccionaron con diferentes solventes, las fracciones de interés se purificaron mediante cromatografía de capa fina y cromatografía de columna en sílica gel.

\section{Evaluación de la actividad antimicrobiana}

Se determinó la actividad antibacterial del extracto crudo de caldo y de la biomasa a una concentración de $2 \mathrm{mg} / \mu \mathrm{L}$ y las fracciones respectivas a $1 \mathrm{mg} / \mu \mathrm{L}$. Se utilizó el ensayo de difusión en gel, con cultivos de 18-24 horas en caldo nutritivo $(\mathrm{pH}$ $6.5+/-0.2$ a $25^{\circ} \mathrm{C}$ ) con los microorganismos Sta- 
phylococcus aureus ATCC 25923, Escherichia coli ATCC 25922 y Bacillus subtilis ATCC, utilizando el método de difusión en agar $(10,11)$.

A los compuestos que resultaron positivos por el método anterior, se les realizó bioautografías, sembrando una placa cromatográfica de $7 \mathrm{~cm}$ de largo x $2.5 \mathrm{~cm}$ de ancho.

Para evaluar la actividad antifúngica se utilizó el método de difusión en pozo (12). Como microorganismo indicador se seleccionó Fusarium oxysporum, por ser de crecimiento rápido y no poseer ciclo sexual definido (10).

\section{Resultados y discusión}

\section{Identificación}

En relación con las características microscópicas de las colonias obtenidas del cultivo de la cepa SPG 321, se observó la presencia de columella elipsoidal, de color café- grisáceo, presencia de hifas cenocíticas con esporangiosporas de pared lisa, elipsoidales de forma alargada y color café ( ver figural a y b).

Frente a la dificultad para la identificación de las más de 50 especies válidas para el género Mucor se evaluó el crecimiento en luz azul como se encontró en el estudio de Fátima Silva y Garre (13) y se observó crecimiento a $37^{\circ} \mathrm{C}$ según literatura (5).

Estos datos permiten realizar la clasificación de la cepa SPG321. Como perteneciente al género Mucor y la presencia de pigmentos naranjas bajo condiciones de luz azul confirman la especie circinelloides (13).
Figura 1.

\section{a. Esporangiosporas}

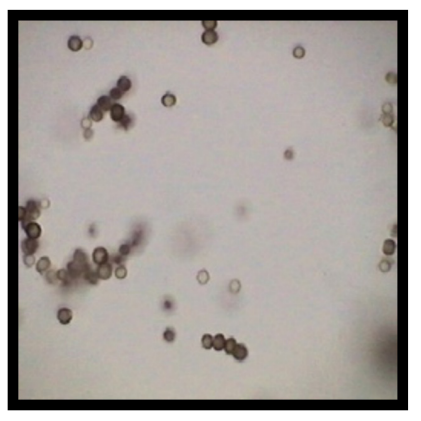

a

\section{b. Columella}

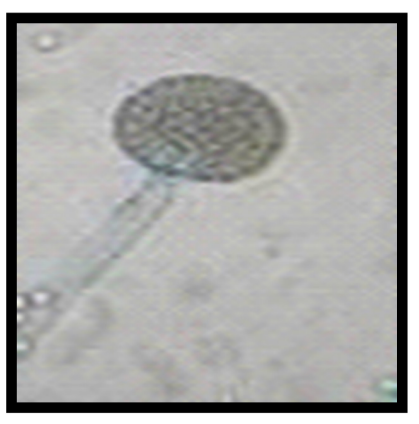

b

Fuente. Elaboración propia.

\section{Preinóculo}

Se manejó un volumen efectivo de trabajo (VET) de $25 \mathrm{ml}$, preparados a partir de $24.5 \mathrm{ml}$ de medio papa-dextrosa-sulfato de amonio (PGS), al que se le agregaron $0,25 \mathrm{ml}$ de una suspensión de conidios a una concentración de 1x 107 conidios/ml. Se incubó a $25^{\circ} \mathrm{C}$ y con agitación a $180 \mathrm{rpm}$; por 3 días conservando la proporción de 1/5 en Erlenmeyer de $125 \mathrm{ml}(7,8)$. 
Figura 2. Preinóculo de la cepa nativa de Mucor circinelloide SPG 321.

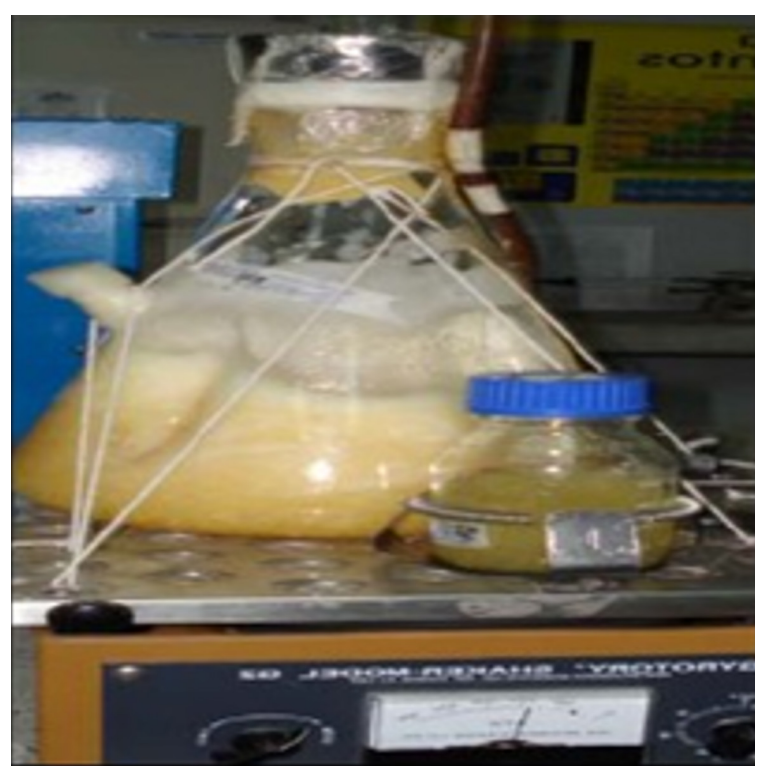

Fuente. Elaboración propia.

\section{Cinética de crecimiento de Mucor circinelloides.}

Para determinar el comportamiento de la cepa nativa SPG 321 en relación con la cinética del microorganismo y su relación con la producción de metabolitos secundarios, se procede al análisis de los parámetros que influyen en el diseño de los procesos de fermentación.

Figura 3. Concentración de biomasa vs tiempo.

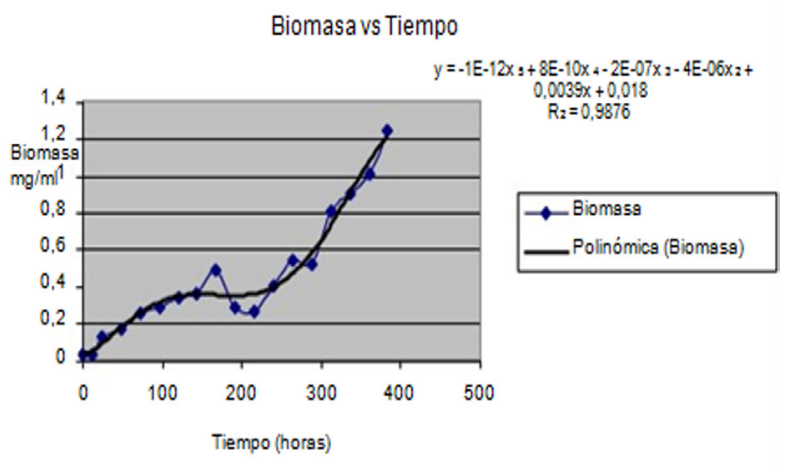

Fuente. Elaboración propia.
La concentración de la biomasa cambió progresivamente en el tiempo, aunque presentó una declinación en la hora 240. Debido a que los nutrientes de primera mano empiezan a agotarse (13) de tal forma que la velocidad de crecimiento declina; la biomasa, alcanzó su máximo peso en la hora 384, debido a la acumulación de productos de almacenamiento como lípidos, polisacáridos y la síntesis de metabolitos secundarios (ver figura 3).

\section{Consumo de sustrato.}

$\mathrm{Al}$ analizar el consumo de sustrato para la cepa nativa SPG 321 de Mucor circinelloides se obtiene la gráfica 4. Se observa una disminución de glucosa en el tiempo, debido a que es usada como fuente de carbono, es decir, disminuye al ser usado como sustrato en la síntesis de nuevo material celular.

Entre las horas 144 y 192 se observa un descenso, con un aumento en la hora 216 , lo que se asocia como una diauxia. Si se tiene en cuenta que el medio PGS contiene dos fuentes de carbono diferentes dextrosa y almidón. Mucor circinelloides utiliza aquella fuente que le proporciona mayor rendimiento energético en este caso la glucosa. Cuando ésta se acaba hay un tiempo de adaptación hacia el segundo substrato que a continuación es consumido, en este caso el almidón.

El rendimiento y velocidad de crecimiento es mayor para el primer substrato que para el segundo.

Figura 4. Consumo de glucosa.

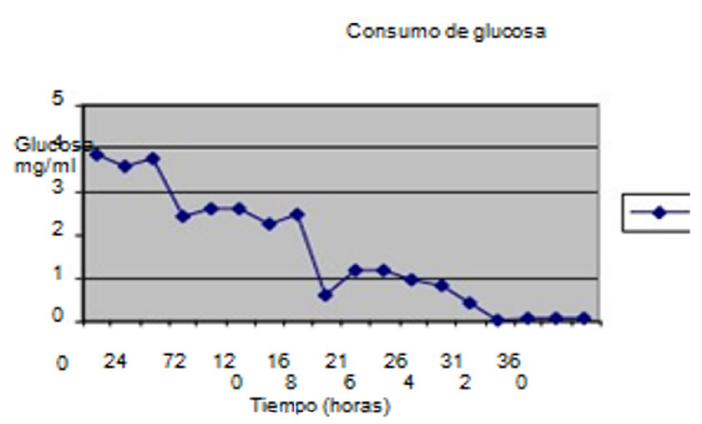

Fuente. Elaboración propia. 
La cepa nativa, de Mucor circinelloides SPG 321 presentó una curva típica de desarrollo; al inocularse en el medio líquido PGS, la tropofase de acuerdo con la concentración celular se inició a las 72 horas, por división celular y formación de nuevo protoplasma a partir de los componentes del medio PGS. La declinación se presentó a partir de la hora 168 debido a que los nutrientes sufren pérdida en la concentración.

La fase conocida como idiofase, se presentó a partir de las 168, horas, para Mucor circinelloides SPG 321, y culminó a las 264 horas día 11.

Figura 5. Tropofase de la cepa SPG 321 de Mucor circinelloides.

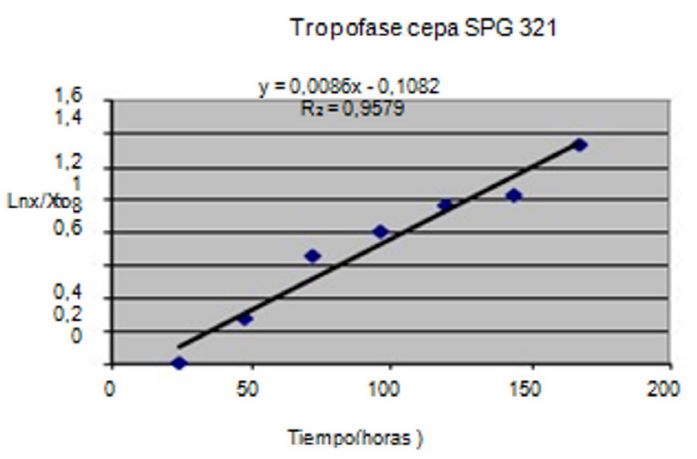

Fuente. Elaboración propia.

La fase de latencia, para la cepa nativa SPG 321 de Mucor circinelloides termina a las 48 horas e inicia la tropofase, una vez se adapta a las condiciones de crecimiento, proporcionadas, por el medio PGS. El crecimiento de la biomasa es descrito cuantitativamente por unidad de tiempo en una gráfica semilogarítmica y culmina a las 168 horas como lo muestra la figura 5 .

Figura 6. Comparación de biomasa y glucosa vs tiempo.

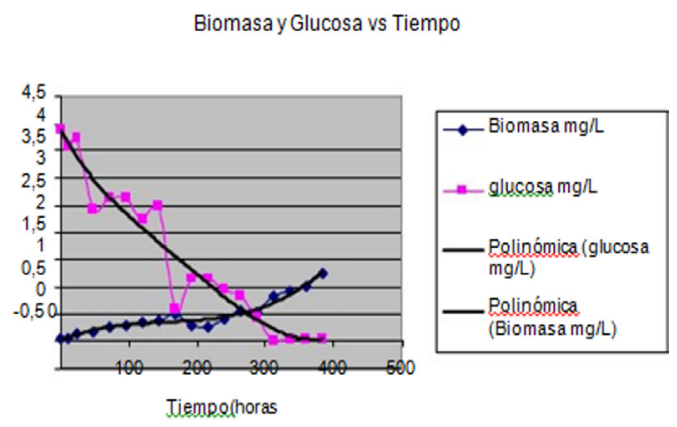

Fuente. Elaboración propia.

\section{Fases de crecimiento cepa nativa SPG $321 \mathrm{Mu}$ - cur circinelloides}

En relación con la cinética, se encontró que la cepa nativa presentó una curva típica de desarrollo al inocularse en el medio líquido PGS, (figura 7) la tropofase inició a las 72 horas y culminó a la hora 168, la idiofase finalizó en la hora 264 día 11 y se tomó éste como día clave para la finalización de la fermentación.

Figura 7. Concentración celular de la cepa nativa SPG 321 de Mucor circinelloides.

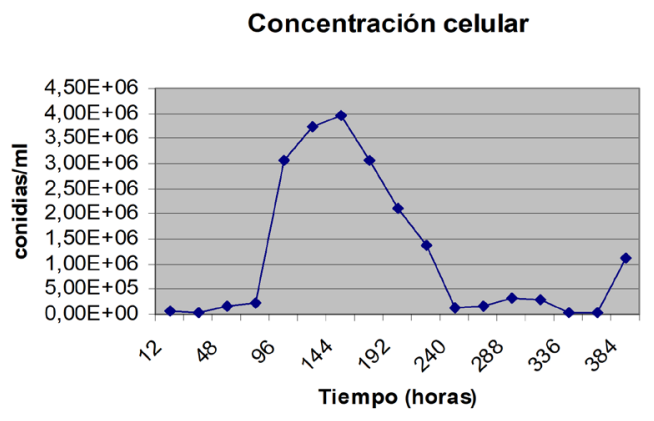

Fuente. Elaboración propia.

\section{Determinación de parámetros cinéticos}

Los parámetros, a determinar en este estudio fueron, $\mu \mathrm{x}$ velocidad específica de crecimiento (h-1), $\mathrm{Y} x / s$ rendimiento de biomasa a partir de sustrato (g/g), td tiempo de duplicación (h) y productividad (gL-1/h). Para determinar los parámetros cinéticos en la fermentación llevada a cabo en un biorreactor de1, 5L, se aplicó la cinética de orden 1 con un R2 $=0,9579$.

Figura 8. Velocidad especifica de crecimiento de Mucor circinelloides en biorreactor.

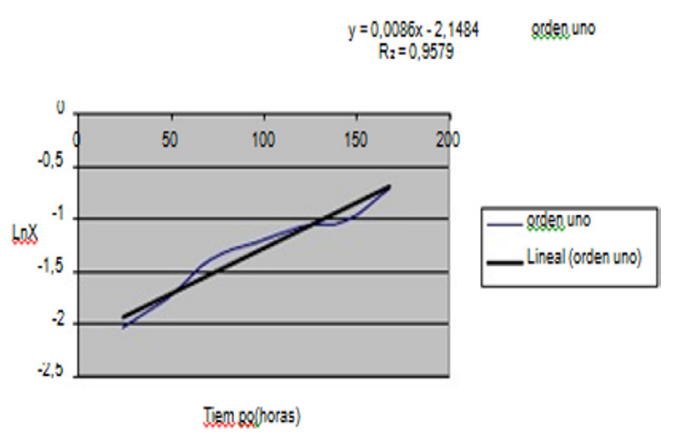

Fuente. Elaboración propia. 


\section{Determinación de parámetros cinéticos}

Los parámetros, a determinar en este estudio fueron, $\mu \mathrm{x}$ velocidad específica de crecimiento (h-1), $\mathrm{Y} \mathrm{x} / \mathrm{s}$ rendimiento de biomasa a partir de sustrato (g/g), td tiempo de duplicación (h) y productividad (gL-1/h). Para determinar los parámetros cinéticos en la fermentación llevada a cabo en un biorreactor de1, 5L, se aplicó la cinética de orden 1 con un R2 $=0,9579$.

Tabla 1. Parámetros cinéticos.

\begin{tabular}{|l|l|l|}
\hline ux & 0,0086 & $\mathrm{mg} / \mathrm{h}-1$ \\
\hline Td orden uno & 80,5985049 & $\mathrm{~h}$ \\
\hline Mmax & 0,86550113 & $\mathrm{mg} / \mathrm{h}$ \\
\hline Ks & 19,5689804 & $\mathrm{~h}-1$ \\
\hline Yx/s & 0,18719512 & $\mathrm{mg} / \mathrm{mg}$ \\
\hline Productividad & 0,00255833 & $\mathrm{mg} / \mathrm{Lh}-1$ \\
\hline
\end{tabular}

Fuente. Elaboración propia.

Analizando el dato obtenido se concluye que la velocidad máxima de crecimiento es baja, debido a las condiciones de fermentación, ya que la cepa de Mucor circinelloides necesita energía adicional para degradar substratos de cadena larga, como es el almidón de papa, presente en el medio.

Comparado con lo reportado por la literatura; para Mucor hiemales, que tiene una $\mu \max$ de 0,17 , en comparación con $0,0086 \mathrm{mg} / \mathrm{h}-1$ este valor confirma que Mucor, estaba en condiciones de estrés, lo cual se deduce, teniendo en cuenta que, en la tropofase, la prioridad es el aumento de biomasa, cuando los microorganismos se cultivan en un medio rico, balanceado de nutrientes

Los metabolitos secundarios tienden a ser biosintetizados después de que la tasa de crecimiento ha cesado. Se desconoce la función del metabolito secundario, pero la bioactividad de estos compuestos ha sido explotada entre otras aplicaciones industriales como medicamentos, venenos, agentes saborizantes (14).

\section{Compuestos químicos aislados}

Del extracto de caldo fracción AcOEt se obtuvieron dos fracciones $\mathrm{CH} 2 \mathrm{Cl} 2$ y PETROL. La biomasa se trabajo en dos fracciones $\mathrm{CHCl} 3$ y PETROL y se aisló un cristal en forma de agujas denominado M.c B3, al que se le realizó análisis de RMN1 H y CG-EM. Por las características del espectro este compuesto se asemeja a un esterol, se reporta la presencia de los ácidos grasos: palmítico, palmitoléico, esteárico y oleico, resultados dados por la técnica de gases acoplado a masas CG-EM, que confirman la importancia de Mucor circinelloides y su potencial como hongo oleaginosos, reportado por su uso comercial en la producción de aceite rico en ácidos grasos insaturados $(15,16)$.

\section{Evaluación antimicrobiana}

Se determinó la actividad antibacterial de la fracción PETROL y $\mathrm{CHCl} 3$ Los datos encontrados para estas dos fracciones se consignan en la tabla 2. Se consideraron indeterminados los halos con diámetro de 13-14 $\mathrm{mm}$ y resistentes los menores o iguales a $12 \mathrm{~mm}$ (17-19). La fracción $\mathrm{CHCl} 3$ no mostró actividad inhibitoria para los microorganismos grampositivos Staphylococcus aureus ATCC 25923 y Bacillus subtillis (ATCC 25921), indeterminado para Eschericha coli (ATCC 25922).

Tabla 2. Actividad antibacteriana en las fracciones PETROL y $\mathrm{CHCl} 3$ de biomasa.

\begin{tabular}{|l|l|l|l|}
\hline \multicolumn{4}{|c|}{ DIÁMETRO PROMEDIO DE HALOS DE } \\
INHIBICIÓN EN mm (milímetros) A LAS 24 \\
HORAS 1 $\mu / \mathrm{L}$ \\
\hline BACTERIA \\
\begin{tabular}{|l|l|l|} 
FRACCIONES \\
Fracción Petrol
\end{tabular} & S. aureus & B. subtilis & E. Coli \\
\hline Control positivo & $12 \mathrm{~mm}$ & $13 \mathrm{~mm}$ & $<5.0 \mathrm{~mm}$ \\
\hline Fracción CHCL3 & $22 \mathrm{~m} \cdot \mathrm{m}$ & $30 \mathrm{~mm}$ & $35 \mathrm{~mm}$ \\
\hline Control positivo & $28 \mathrm{~m} \cdot \mathrm{m}$ & $25 \mathrm{~mm}$ & $13.5 \mathrm{~mm}$ \\
\hline
\end{tabular}

Fuente. Elaboración propia. 
Figura 9. Pruebas antibacterianas A. S. aureus B. B. subtilis y C. E. coli
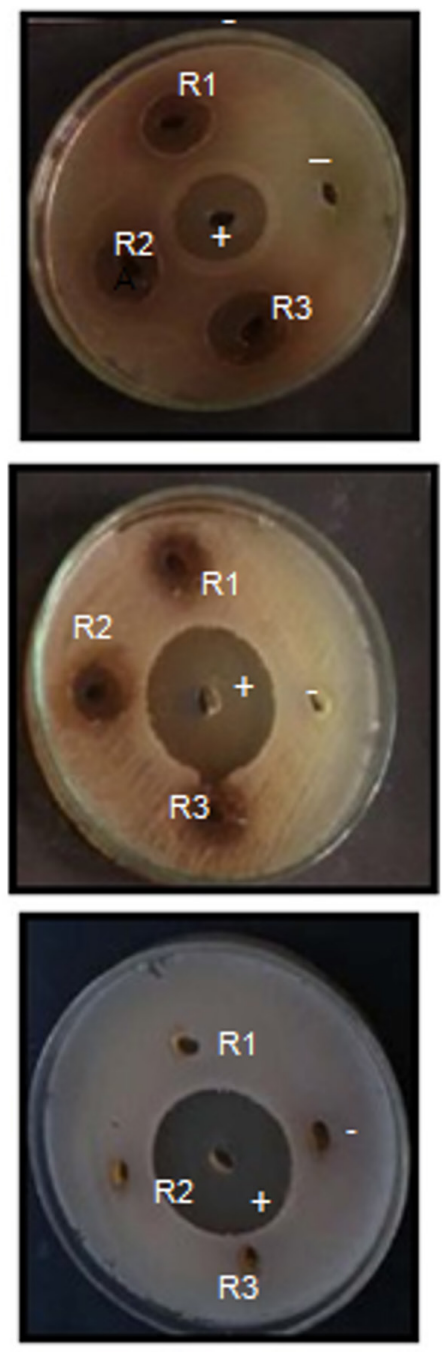

Fuente. Elaboración propia.

Los resultados permiten concluir que la fracción $\mathrm{CHCl} 3$ presentó actividad inhibitoria contra Staphylococcus aureus ATCC 25923 activa y contra Bacillus subtilis (ATCC 25921), aunque se trata de fracciones orgánicas cuya composición se desconoce. La fracción $\mathrm{CHCl} 3$ que dio positiva por el método anterior, se le realizó contraplacas por la técnica de la bioautografías con zona de inhibición.

Se midieron los frentes de las bandas observadas de cada cromatograma y se determinó un Rf para la fracción $\mathrm{CHCl} 3$ (20-21), en medio con suspensión de $S$. aureus correspondiente a la región de macrólidos. Los extractos totales de caldo de cul- tivo, biomasa y sus correspondientes fracciones y compuesto M.c B3 de la fermentación, probados frente a Fusarium oxysporum no presentaron actividad alguna.

\section{Conclusión}

Los resultados obtenidos muestran, que la fracción $\mathrm{CHCl} 3$ en biomasa presenta actividad inhibitoria para los microorganismos grampositivos Staphylococcus aureus ATCC 25923 y Bacillus subtilis (ATCC 25921), lo que sugiere una fuente de productos con potencial biotecnológico y colocan a la cepa nativa SPG 321 de Mucur circinelloides como un microorganismo promisorio.

\section{Referencias}

1. Álvarez M, Escobar F, Gastv F, Mendoza H, Repizzo A, Villareal H. Ecosistemas Terrestres. Bosque Seco Tropical, en Informe nacional sobre el estado de la biodiversidad. Bogotá, Colombia: Eds. María Elefi Chaves y Natalia Arango; 1997. $129 \mathrm{p}$.

2. Cabrera C, Chitiva A. Aislamiento e identificación de hongos filamentosos del suelo del páramo de Guasca (Colombia) en zona de vegetación de frailejones. [Trabajo de grado]. Bogotá: Pontificia Universidad Javeriana, Facultad de Ciencias; 2001.

3. Gilman J. Manual de los Hongos del Suelo. México: Comp. Ed. Continental; 1963.572 p.

4. Samsong R, Hoekstra E, Van OC. Introduction to food-borne fungi. C.B.S. Institute of the royal netherlands academy of arts and sciences; 1981. 66-67 p.

5. Weitzman I, Whittier J, McKitrich, Della-Latta P. Zygospores: the last word in identification of rare or atypical zygomycetes isolated; 1995.

6. Velez P, Posada F, Marín P, González M, Osorio E, Bustillo A. Técnicas para el control de calidad de formulaciones de hongos entomopatógenos. CENICAFE; 1997: 10.

7. Demain AL. Fungal secondary metabolism: regulation and functions. In: Sutton B (ed). A century of mycology. USA: Cambridge University Press; 1996. 233-254 p.

8. Daza A. Capacidad Biotransformadora y actividad antimicrobiana de los metabolitos secundarios del Aspergillus niger 511. [Trabajo de grado] Bogotá: Pontificia Universidad Javeriana, Facultad de Ciencias; 2001. 64 p.

9. Medina Buelvas A. Evaluación de la capacidad biotransformadora de la cepa nativa de Fusarium oxysporum sobre el 
beta-D- glucopiranosil-ester del ácido(-)17-(beta-glucopiranosiloxil)-16-ol-kauran 19-oico e identificación de metabolitos secundarios. [Trabajo de grado] Bogotá: Pontificia Universidad Javeriana, Facultad de Ciencias; 2001. 66 p.

10. Koneman E y otros. Diagnóstico Microbiológico. Editorial Panamericana; 1999. 612-613 p.

11. NCCLS-National Comité for Clinical laboratory. Performance Standars for Antimicrobial Disk Susceptibility Tests; Approved Standard. Document M2-A8. Nacional Comité for Clinical Laboratory Standard; 2003. 23(1).

12. Becerra N, Segura I. Determinación de la actividad antimicrobiana de las partes aéras (hojas y corteza) de Bursera tomentosa contra algunos fitopatogenos y patógenos del hombre. [Trabajo de grado]. Bogotá: Pontificia Universidad Javeriana, Facultad de Ciencias; 2003.

13. Silva Torres-Martínez, Garre. Distinct white-collar-1 genes control specific light responses in Mucor circinelloides. Mol Microbiol. 2006; 61:1023- 27.

14. Bentley R. Microbial secondary metabolites play important roles in medicine: prospects to discovery of new drugs. Perspect Biol Med. 1997; 40: 364-394

15. Wynn J, Kendrick A, Ratledge C. Sesamol as a inhibitor of growth and lipid metabolism in Mucor circinelloides via its action on malic enzyme. Lipids; 1997 (32): 605-61

16. Antczak T, Hiler D, Krystynowicz A, Szczesna M, Bielecki S, Galas E. Activity of immobilized in situ intracellular lipase from Mucor circinelloides and Mucor racemosus in synthesis of sucrose esters. In: Bielecki S, Tramper J, Polak J, (editors). Progress in biotechnology [vol. 17]. Amsterdam: Elsevier; 1999. 221-8 p.

17. Pinilla B Gladys, Chavarro P Bibiana, Moreno A Natalia, Navarrete O Jeannette, Muñoz M Liliana. Determinación de los genes, 16S ADNr, polA, y TpN47, en la detección de Treponema pallidum subsp. pallidum para el diagnóstico de sífilis congénita. Nova. 2015; $13(24$ ): 17-25.

18. Corrales Lucia Constanza, Antolinez Romero Diana Marcela, Bohórquez Macías Johanna Azucena, Corredor Vargas Aura Marcela. Bacterias anaerobias: procesos que realizan y contribuyen a la sostenibilidad de la vida en el planeta. Nova. 2015; $13(24)$ ) 55-81.

19. 19. Carrero Sandra Helena Suescún, HerediaMontoya Dina Paola, Bolaños Yoryany Mulato, Medellín Martín Orlando Pulido. Seroprevalencia de infección por Leptospira y factores de riesgo en estudiantes de una universidad de Colombia. Nova. 2017; 15 ( 27 ): 131-138.

20. Zuluaga Martha, Robledo Sebastian, Osorio-Zuluaga German A, Yathe Laura, Gonzalez Diana, Taborda Gonzalo. Metabolomics and pesticides: systematic literature review using graph theory for analysis of references. Nova. 2016; 14( 25 ): 121-138.

21. Ávila de Navia Sara Lilia, Estupiñán-Torres Sandra Mónica, Díaz González Liliana. Calidad bacteriológica del agua Vereda El Charco, San Miguel de Sema, Boyacá- Colombia. Nova. 2016; 14( 25 ): 139-145. 